\title{
Cross-modality effects upon choice reaction time
}

LENORE K. MORRELL, DIVISION OF NEUROLOGY, STANFORD UNIVERSITY SCHOOL OF MEDICINE, PalO Alto, Cal. 94304

Facilitation of reaction time by a second stimulus in another sense modality than the initial signal for response extended over a longer range of interstimulus intervals when the presented sequence was visual-auditory as compared with auditory-visual. The expected difference between visual and auditory reaction times was diminished or eliminated with paired bi-modal stimuli.

Reaction time (RT) to a visual signal may be facilitated when it is followed by an auditory stimulus $20-120 \mathrm{msec}$ afterwards; faster responses occurred at the shorter interstimulus intervals. The effect was observed when every trial in the series contained the visual reaction signal (Morrell, 1967) and when signal discrimination was required (Morrell, in press). In this report, an auditory-visual sequence will be compared with a visual-auditory one. Since the auditory and visual modalities differ in neural latency, a difference in the time course of the intersensory effects would be predicted between the two sequences.

In the present series, the reaction signal did not occur on every trial. This condition minimized S's tendency to respond to any stimulus, and ensured that the reaction process depended upon registration of the signal defined as the reaction stimulus.

Method. The apparatus and general procedure were identical in all respects to that previously described (Morrell, in press). In the visual-auditory sequence series the S's task was to respond as quickly as possible to a $10 \mathrm{msec}$ flash by depressing a small switch lightly taped to his right hand. This occurred on seven out of eight trials; however, in six of these a click was presented afterwards at interstimulus intervals ranging from $20-120 \mathrm{msec}$. In one out of every eight trials the click stimulus was presented alone, and to this event the $S$ was instructed to withhold motor response. A session consisted of 288 trials with 36 presentations of each of the eight stimulus configurations (i.e., flash alone, flash-click pairs separated by $20,40,60,80,100,120$ msec, and click alone). These were given in quasi-random order, with the constraint that no configuration was repeated on the immediately following trial.

The auditory-visual sequence required the $S$ to respond manually only to trials containing a click stimulus and to withhold response to flash when it occurred alone. The experimental sequencing was identical to the visualauditory one described above, except that click preceded flash on all paired trials.

A warning tone preceded each trial by $1-2 \mathrm{sec}$.

Records were obtained from four Ss, all males from 21-32 years of age. Two of these received the flash-click series first, while the other two received the click-flash series first.

Results. Figure 1 presents the data for each $S$ under each condition. Each point represents the median reaction time of 36 trials of a given type. Two-way analysis of variance, performed for the grouped data from each series separately, showed that the stimulus conditions caused significant variation in RT ( $p<.01$ for the flash-click sequence, $p<.05$ for the click-flash sequence). Figure 1 also shows the slope of the function $b$ obtained from linear regression analysis relating reaction times to interstimulus interval in each instance; all $\mathrm{p}$ values were less than .05 for these slope values except for the data obtained from $S_{3}$ for the click-flash series. Thus with paired stimuli in both the visualauditory or auditory-visual sequence, where in each case the task of the $S$ is to respond to the first stimulus, average reaction times are faster at the shorter interstimulus intervals.

RTs to each of the flash-click pairs ranging from $20 \mathrm{msec}$ to 120 msec interstimulus interval were significantly faster than RT to a single flash. However, in the click-flash series only paired stimuli separated by $40 \mathrm{msec}$ or less yielded significantly faster RTs than did the single click. These findings were evaluated by the Tukey studentized range test; $\mathrm{p}$ values ranged from .01 to .05 .

The difference in RT between the visual and the auditory stimulus when the single presentations were considered were as expected; auditory RT is faster than visual RT. However, the expected shorter RTs to click were not always observed when it was followed by flash. To evaluate this, for each S the set of RTs to a flash-click pair at a given interstimulus interval was compared with the set of RTs to a click-flash pair at the same interval with the Mann-Whitney U-test. This was done for each $S$ for each stimulus condition; the results are summarized in Table 1 . In all cases where a significant difference was obtained, RTs were faster when click was the initial signal. At the $20 \mathrm{msec}$ interstimulus interval, three out of four of the Ss showed significantly faster RTs to the click-flash sequence than to the flash-click sequence. At intervals between $40-80 \mathrm{msec}$, only one $S$ showed this difference (S2). At 100 and $120 \mathrm{msec}$ intervals, two Ss showed faster RTs to the click-flash pair than to the flash-click pair. For the single stimulus condition, three of the four Ss showed significantly faster response to click than to flash. The U-test does not evaluate the relative magnitude of these differences. Therefore, for each $S$ a score was obtained by taking the difference between his median RT to flash and median RT to click at each interstimulus interval, as well as for the single stimulus condition (scores shown in Table 1). A two-way analysis of variance test (for four $S$ s and seven difference scores per S) showed that there were significant effects of interstimulus interval upon the difference scores $(p<.05)$. Tukey pair-wise tests then indicated that the RT difference at the single stimulus condition was reliably greater than the differences observed for each of the following interstimulus intervals: $20 \mathrm{msec}, 60 \mathrm{msec}, 80 \mathrm{msec}, 100 \mathrm{msec}$, (all p values $<.05$ ).

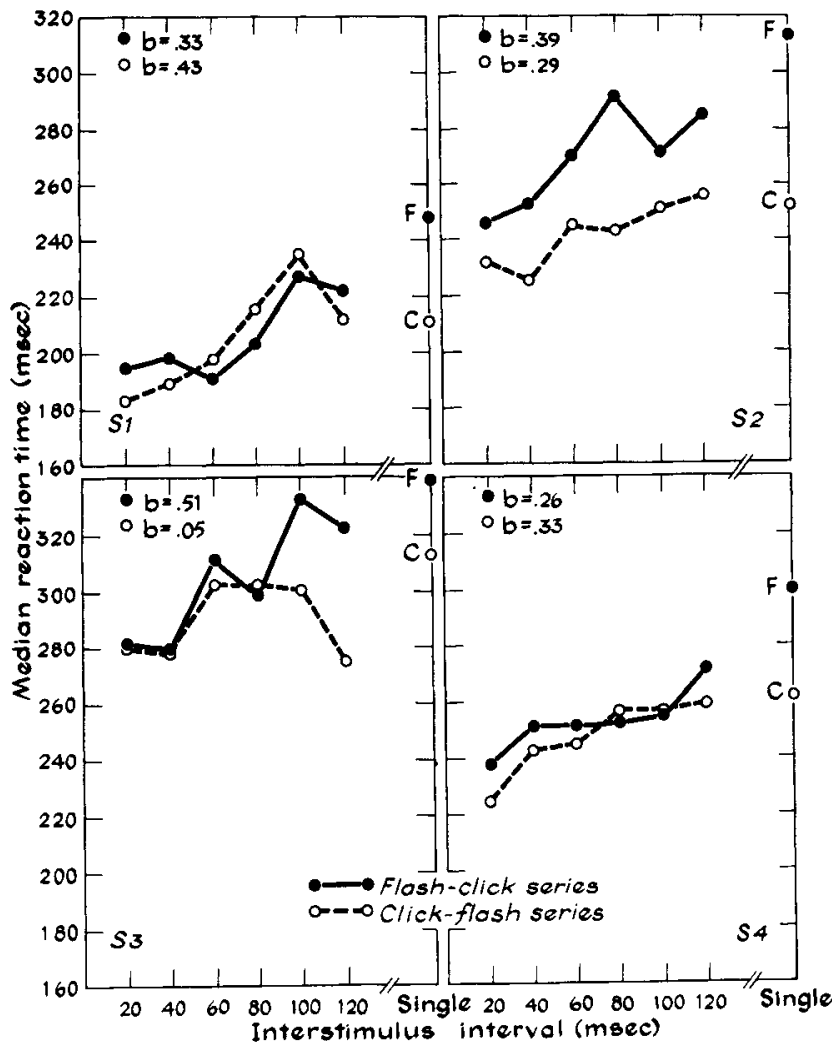

Fig. 1. Median RT for each $\mathrm{S}$ for each condition in both series. Each point represents median of 36 observations. S1, S2, S3, S4 are individual Ss. The b values in the upper left corner of each graph are the slopes ot the tunction relating $\mathrm{RT}$ to interstimulus interval obtained from linear regression analysis; these were calculated for each S only from the paired stimuli data. RT to single flash and single click shown at extreme right of each graph. 
Table 1

Differences Between RTs in Flash-click Series vs Click-flash Series for Each of Four Ss

INTERSTIMULUS INTERVAL

\begin{tabular}{lccccccc} 
& $20 \mathrm{msec}$ & $40 \mathrm{msec}$ & $60 \mathrm{msec}$ & $80 \mathrm{msec}$ & $100 \mathrm{msec}$ & $120 \mathrm{msec}$ & Single stimulus \\
\hline S1 & $12^{*}$ & 10 & -7 & -7 & -9 & 12 & $39^{*}$ \\
S2 & $15^{*}$ & $28^{* *}$ & $25^{*}$ & $49^{* *}$ & $20^{*}$ & $29^{* *}$ & $62^{* *}$ \\
S3 & 0 & 0 & 8 & -4 & $31^{*}$ & $48^{* *}$ & 26 \\
S4 & $14^{*}$ & 9 & -7 & -5 & -1 & 13 & $39^{*}$ \\
\hline
\end{tabular}

Note. Entries are differences between median $R T$ values for each interstimulus interval and single flash vs single click. Flash-click always greater than click-flash unless entry is preceded by (-) which signifies that click-flash $R T$ was greater. $S_{1}, S_{2}, S_{3}, S_{4}$ are individual Ss.

${ }^{*} p<.05,{ }^{* *} p<.01$, one tailed, Mann-Whitney U-tests.

Discussion. RTs to paired stimuli with a visual reaction signal followed by an auditory stimulus are faster than RT to single flash over the interstimulus range 20-120 msec. However, with an auditory reaction signal followed by a visual stimulus, the temporal range of facilitation is shorter than with the visual-auditory sequence.

Neural responses to auditory stimulj are faster than to visual stimuli. As an approximation, the average figure of $30 \mathrm{msec}$ difference will be used for further evaluation of the present data. This figure was used by Davis (1957) and is consistent with electrophysiological measurements at primary cortical areas (Bartley, 1934; Kemp, Coppée, \& Robinson, 1937) and motor cortex (Buser \& Imbert, 1961). Using the $30 \mathrm{msec}$ estimate, the effective interstimulus intervals as approximated by physiological time are quite different from clock time. For the clock time interstimulus intervals of 20 , $40,60,80,100$, and $120 \mathrm{msec}$, the physiological intervals for the flash-click series may be approximated as $-10,10,30,50,70$, and $90 \mathrm{msec}$ while those for the click-flash sequence would be approximated by $50,70,90,110,130$, and $150 \mathrm{msec}$. In the flash-click sequence at $20 \mathrm{msec}$ clock time separation, the auditory stimulus may precede the visual stimulus in neural latency; hence the -10 approximate physiological interval. The temporal course of the intersensory effect is more similar for the two sequences when one takes neural latency differences into account.

While comparison of RT to flash alone with RT to click alone showed response to the auditory stimulus was faster by an average of $40 \mathrm{msec}$, the expected differences between these two modalities tended to be diminished or wiped out with cross-modal pairing. Hershenson (1962) and Adams \&
Chambers (1962) noted a similar effect under quite different experimental conditions.

ADAMS, J. A., \& CHAMBERS, R. W. Response to simultaneous stimulation of two sense modalities. J. exp. Psychol., 1962, 63, 198-206.

BARTLEY, S. H. Relation of intensity and duration of brief retinal stimulation by light to the electrical responses of the optic cortex of the rabbit. Amer. J. Physiol., 1934, 108, 397-408.

BUSER, P., \& Imbert, M. Sensory projections to the motor cortex in cats: A microelectrode study. In W. A. Rosenblith (Ed.), Sensory communication. New York: MIT Press and John Wiley \& Sons, Inc., 1961. Pp. 607-624.

DAVIS, R. The human operator as a single channel information system. Quart. J. exp. Psychol. 1957, 9, 119-129.

HERSHENSON, M. Reaction time as a measure of intersensory facilitation. $J$. exp. Psychol., 1962, 63, 289-293.

KEMP, E. H., COPPÉE, G. E., \& ROBINSON, E. H. Electric responses of the brain stem to unilateral auditory stimulation. Amer. J. Physiol., 1937, 120, 304-322.

MORRELL, L. K. Intersensory facilitation of reaction time. Psychon. Sci., $1967,8,77.78$

MORRELL, L. K. Temporal characteristics of sensory interaction in choice reaction times. $J$. exp. Psychol, in press.

\section{NOTE}

1. Supported by NASA Grant NsG 215-62. The aid of Dale Borglum in statistical evaluation is gratefully acknowledged. 